\title{
Dual function of MyD88 in RAS signaling and inflammation, leading to mouse and human cell transformation
}

\author{
Isabelle Coste, ${ }^{1,2}$ Katy Le Corf, ${ }^{1}$ Alain Kfoury, ${ }^{1}$ Isabelle Hmitou, ${ }^{3}$ Sabine Druillennec, ${ }^{3}$ \\ Pierre Hainaut, ${ }^{2}$ Alain Eychene, ${ }^{3}$ Serge Lebecque, ${ }^{1}$ and Toufic Renno ${ }^{1,4}$ \\ ${ }^{1}$ CNRS UMR5201, University of Lyon, Centre Léon Bérard, Lyon, France. 2International Agency for Research on Cancer, Lyon, France. \\ 3Institut Curie, INSERM U1021-CNRS UMR3347, Orsay, France. ${ }^{4}$ Schering-Plough, Dardilly, France.
}

\begin{abstract}
Accumulating evidence points to inflammation as a promoter of carcinogenesis. MyD88 is an adaptor molecule in TLR and IL-1R signaling that was recently implicated in tumorigenesis through proinflammatory mechanisms. Here we have shown that MyD88 is also required in a cell-autonomous fashion for RAS-mediated carcinogenesis in mice in vivo and for MAPK activation and transformation in vitro. Mechanistically, MyD88 bound to the key MAPK, Erk, and prevented its inactivation by its phosphatase, MKP3, thereby amplifying the activation of the canonical RAS pathway. The relevance of this mechanism to human neoplasia was suggested by the finding that MyD88 was overexpressed and interacted with activated Erk in primary human cancer tissues. Collectively, these results show that in addition to its role in inflammation, MyD88 plays what we believe to be a crucial direct role in RAS signaling, cell-cycle control, and cell transformation.
\end{abstract}

\section{Introduction}

In order to insure detection of a broad variety of pathogens, the innate immune system has evolved a strategy to recognize a limited number of conserved microbial features termed pathogenassociated microbial patterns (PAMPs). PAMPs are shared by members of particular classes of microbes and are recognized by evolutionarily conserved pattern-recognition receptors (PRR). An extensively studied family of PRR is the TLR family (1).

TLRs, which lack catalytic domains, are connected to the cellsignaling machinery via intracellular adaptor molecules. The first such adaptor molecule to be discovered was MyD88. In addition to its C-terminal Toll/IL-1R interacting resistance (TIR) domain, MyD88 has an N-terminal death domain (DD), which recruits downstream signaling molecules (2). IL-1 is another key inflammatory mediator that utilizes MyD88 for its signaling.

Inflammation is recognized as a promoter of carcinogenesis (3), and recent studies point to a role for MyD88 in the protumorigenic inflammatory response. For instance, RakoffNahoum and Medzhitov have crossed MyD88-deficient mice to $A P C^{\mathrm{min} /+}$ mice, which carry a germ-line mutation in the tumor suppressor gene Apc, and showed that absence of MyD88 in this model resulted in a decrease in the number of polyps in the small intestine and in the colon, associated with substantially increased survival (4). A number of inflammation-related genes were shown to be decreased in these mice, prompting the authors to conclude that MyD88 signaling downstream of members of the TLR and IL-1R family has a critical role in intestinal tumorigenesis (4). Using 2 distinct chemical carcinogenesis models (DMBA/TPA and MCA), Swann et al. (5) confirmed the role of MyD88 in inflammation-induced carcinogenesis. Interestingly, the authors discussed the possibility that MyD88 may act intrinsically to facilitate fibroblast and epithelial cell

Authorship note: Isabelle Coste and Katy Le Corf contributed equally to this work. Conflict of interest: The authors have declared that no conflict of interest exists. Citation for this article: J Clin Invest. 2010;120(10):3663-3667. doi:10.1172/JCI42771. transformation (5). Here we show that in addition to its role in inflammation, MyD88 indeed plays a cell-autonomous role in Ras-mediated transformation via its interaction with the canonical MAPK Erk.

\section{Results and Discussion}

To investigate the molecular mechanisms through which MyD88 mediates tumorigenic signaling, we subjected WT and MyD88mice to a 2-stage, RAS-dependent skin carcinogenesis protocol using 7,12-dimethylbenz(a)anthracene (DMBA) as an initiator and 12-O-tetradecanoylphorbol 13-acetate (TPA) as a promoter (6). Less than 5\% of $M y D 88^{-/-}$mice developed tumors in response to DMBA/TPA treatment, whereas virtually all WT mice developed skin papillomas by the end of the treatment period (Figure $1 \mathrm{~A}$ and Supplemental Figure 1; supplemental material available online with this article; doi:10.1172/JCI42771DS1). This resistance was found not to be solely due to an absence of IL-1 proinflammatory signaling, since $I L-1 R^{-/-}$mice, while less susceptible than WT mice to tumor induction, did readily develop skin tumors in response to DMBA/TPA (Figure 1A). Collectively, these data suggested other, noninflammatory functions for MyD88 in RAS-mediated tumor development.

To address whether MyD88 acts in a cell-autonomous fashion, we generated mouse embryonic fibroblasts (MEF) from WT and $M y D 88^{-/-}$mice. $M y D 88^{-/-}$MEFs were resistant to cell transformation by DMBA/TPA in vitro, as assessed by the focus formation assay (Supplemental Figure 2). MyD88-/- MEFs were similarly resistant to transformation after transfection with Ras ${ }^{\mathrm{V} 12} / \mathrm{Myc}$ (Figure $1 \mathrm{~B}$ and Supplemental Figure 2). This resistance was not due to a general defect in transformation, since $M y D 88^{-/-}$MEFs are readily transformed with SV40 T antigen (7), suggesting a selective role for MyD88 in Ras signaling and transformation. We therefore asked whether MyD88 is involved in the canonical Ras/MAPK signaling pathway. While neither p38 nor Akt phosphorylation was affected by absence of MyD88 (Supplemental Figure 3), Erk MAPK phosphorylation was substantially reduced in MyD88-deficient MEFs 


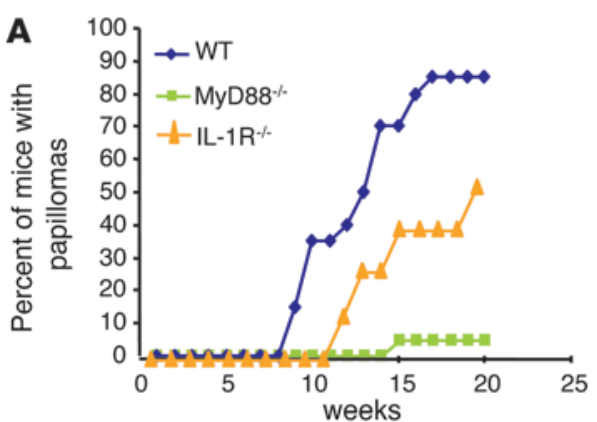

C
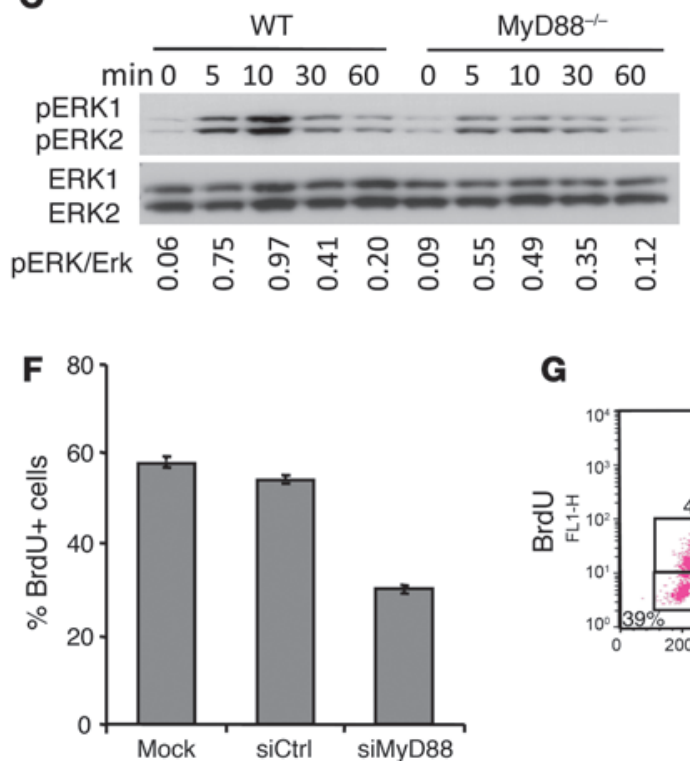
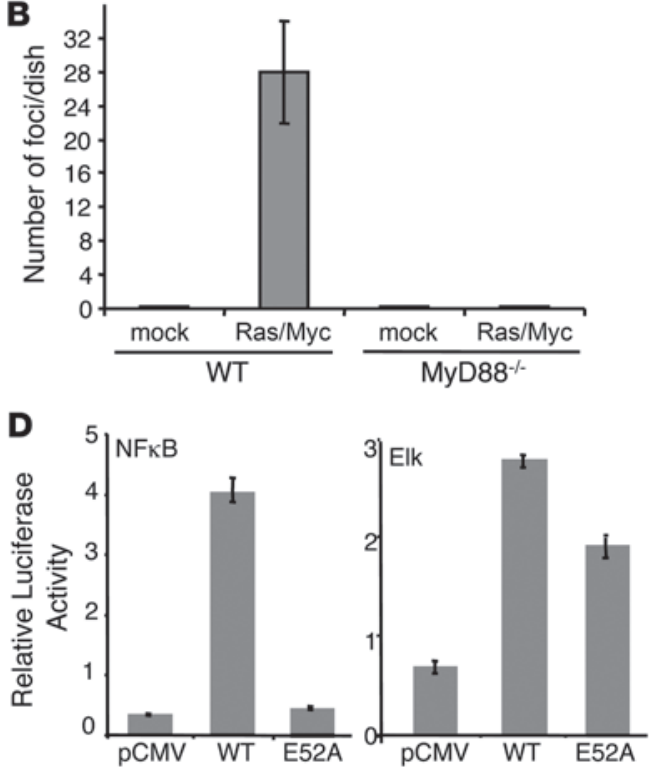

E
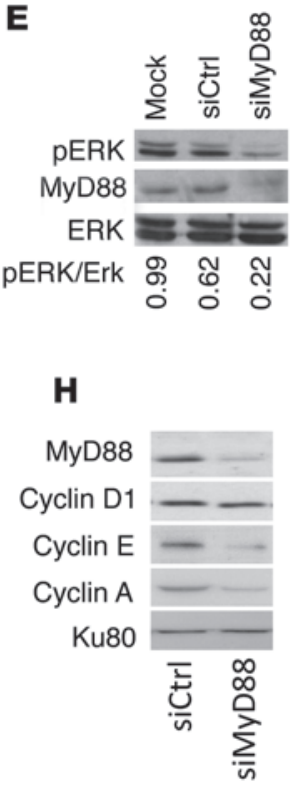

Figure 1

MyD88 is required for Ras transformation, full Erk activation, and cell cycle. (A) Incidence of skin papilloma in WT, MyD88 $8^{-/-}$, and $I L-1 R^{-/-}$mice ( $n=20$ per group) subjected to a 2-stage carcinogenesis (DMBA and TPA). (B) Quantification of foci developed in WT and MyD88 ${ }^{-/-}$MEFs transfected with Ras ${ }^{12}$ and Myc. (C) Erk phosphorylation is reduced in MyD88-- MEFs treated with FGF for the indicated times. Lower panel shows total Erk on the same blots. (D) Luciferase activity from NF-kB luciferase or Elk luciferase in HeLa cells overexpressing WT MyD88, MyD88-E52A, or control plasmid. Values were normalized based on Renilla luminescence. (E-H) A375 melanoma cell line was transfected with control (siCtrl) or MyD88-specific siRNA (siMyD88). (E) Western blot shows reduced constitutive Erk phosphorylation in MyD88 siRNA-treated cells. (F) Decreased percentage of cells incorporating BrdU after a 1-hour pulse. (G) BrdU/propidium iodide profile showing a block in the $\mathrm{G}_{1} / \mathrm{S}$ transition of the cell cycle. Boxes (clockwise starting from lower left) represent populations in $G_{0} / G_{1}, S$, and $G_{2} / M$, respectively. (H) Western blot showing reduced expression of cyclins $A$ and $E$, but not of cyclin D, in MyD88 siRNA-treated cells. Ku80 is a housekeeping protein used as a gel-loading control. Bar graph data represent mean \pm SEM.

treated with FGF (Figure 1C). We then retrovirally transduced $M y D 88^{-/-}$MEFs with MyD88. As shown in Supplemental Figure 4, the expression levels of MyD88 in reconstituted MyD88-/- MEFs were comparable to those of WT MEF. Treatment of these cells with FGF resulted in Erk phosphorylation in WT MEFs and MyD88reconstituted MyD88-/- MEFs to a similar extent (Supplemental Figure 4), confirming that the defect in Ras/MAPK signaling in the MyD88-/ MEFs is solely due to absence of MyD88.

Recently, Loiarro et al. (8) identified the IRAK interaction motif in MyD88 (E52) and showed that a mutant (E52A) MyD88 is unable to interact with IRAK and to activate NF-KB. To ascertain that the role of MyD88 in Ras/MAPK activation is not limited to an autocrine production of NF- $\mathrm{KB}$-dependent factors, we tested the ability of a MyD88-E52A mutant construct to activate the NF- $\mathrm{KB}$ or the Ras/Erk/Elk pathways. We showed that whereas MyD88-E52A completely lost the ability to activate NF- $\mathrm{kB}$, it retained to a large extent its ability to activate the Ras/Erk/Elk pathway (Figure 1D). Interestingly, the fact that MyD88-E52A is less efficient than WT MyD88 in activating this pathway suggests that NF-KB-dependent factors also contribute to cell activation via MyD88.

Having shown that MyD88 is required for tumor initiation both in vitro and in vivo, we wondered whether it is also required for the maintenance of the transformed phenotype in established tumor cell lines. The human melanoma cell line A375 carries, and is dependent on, an activating mutation in the BRAF gene (9). A375 cells thus have a constitutively active MAPK pathway and therefore exhibit a continuously phosphorylated form of Erk (9). Treatment with siRNA against MyD88 decreased the levels of phosphorylated Erk (Figure 1E). This decreased Erk phosphorylation was accompanied by decreased proliferation, as assessed by BrdU incorporation (Figure 1F). 
A

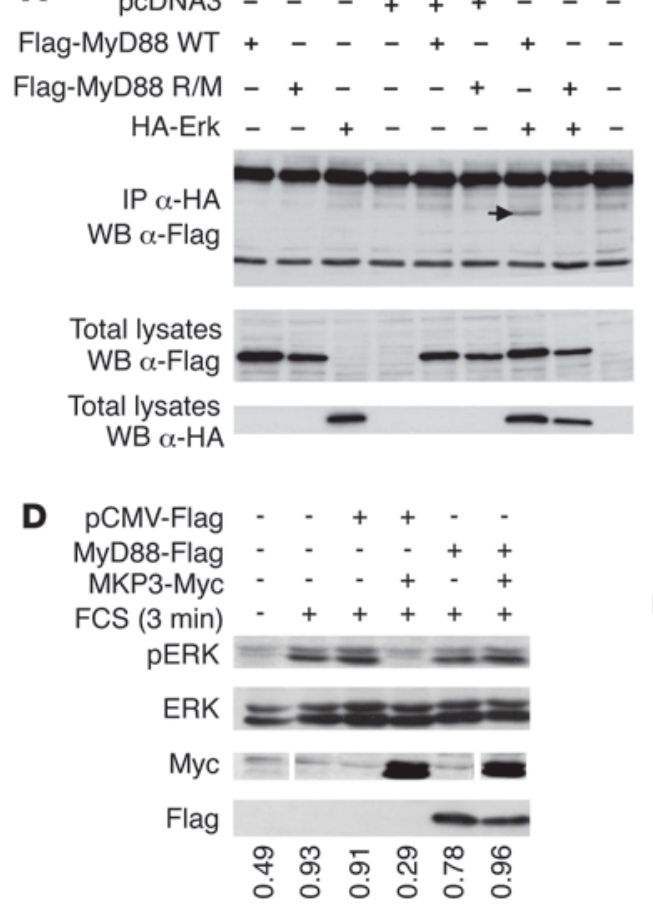

B

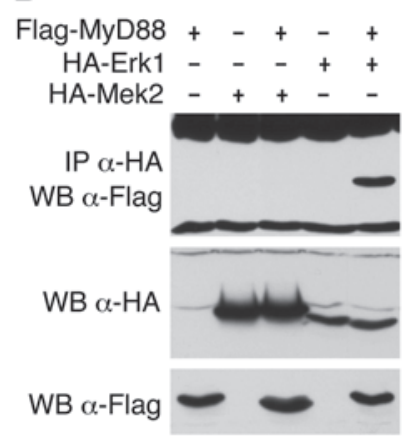

$\mathbf{E}$

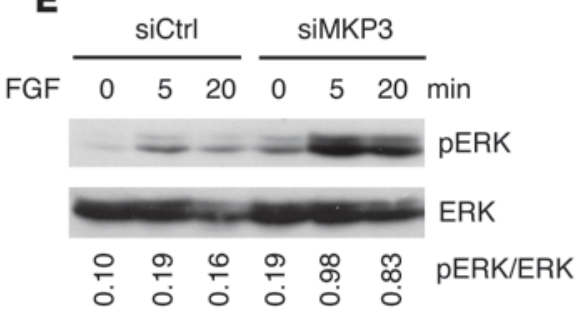

C

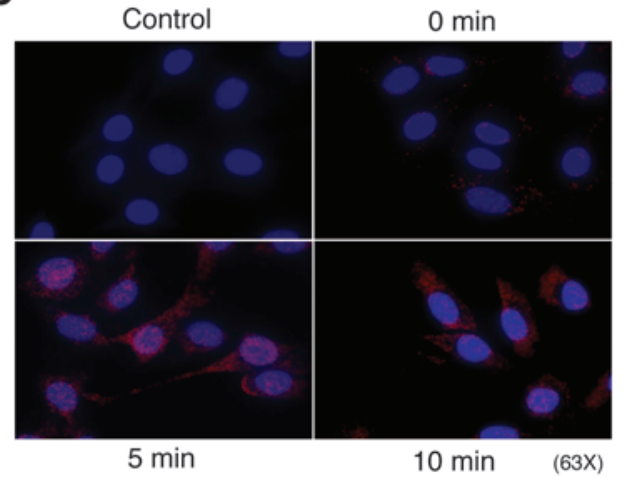

\section{Figure 2}

MyD88 forms a functional complex with Erk and protects it from dephosphorylation by MKP3. (A) MyD88 interacts with Erk via a conserved motif in its death domain. HEK293T cells were transfected with a plasmid encoding HA-Erk and/or Flag-MyD88 (WT), and Flag-MyD88 R/M. Lysates were immunoprecipitated with anti-HA antibody and immunoblotted with anti-Flag antibody (upper panel). Only WT MyD88 interacted with Erk (arrow). (B) MyD88 does not bind to MEK. HEK293 cells were cotransfected with Flag-MyD88 construct and with HA-Erk1 or HA-Mek2. Lysates were immunoprecipitated with anti-HA and immunoblotted with anti-Flag antibody. (C) Interaction between endogenous MyD88 and p-Erk upon cell activation revealed by proximity ligation assay (red dots). B16F10 melanoma cells were starved overnight (0 minutes), then activated with FCS for 5 or 10 minutes. Cells were stained with antibodies to MyD88 and p-Erk followed by the appropriate DNA-linked secondary antibodies according to the Duolink protocol. Control: labeling in absence of primary antibodies. (D) MyD88 inhibits p-Erk dephosphorylation by MKP3. MyD88-Flag, pCMV-Flag, and MKP3-Myc were transfected into HeLa cells as indicated. Cells were starved, then activated with FCS. Western blot analysis was performed on total cell extracts using antibodies to p-Erk, Erk, Flag, and Myc. (E) MKP3 knockdown restores Erk activation in MyD88-/- MEFs. MyD88-deficient MEFs were transfected with control siRNA (siCtrl) or MKP3-specific siRNA (siMKP3), starved, then activated for the indicated times with FGF. Upper panel is a Western blot revealed with anti-p-Erk; lower panel with anti-total Erk.

This reduction was accompanied by a decrease in the number of cells in the $S$ phase and an accumulation in the $G_{0} / G_{1}$ phase, suggesting a block at the $\mathrm{G}_{1} / \mathrm{S}$ transition of the cell cycle (Figure $1 \mathrm{G})$. This block is accompanied by a reduction in the expression of cyclins $\mathrm{E}$ and $\mathrm{A}$, but not of cyclin D (Figure $1 \mathrm{H}$ ), indicating a role for MyD88 in the regulation of proteins controlling the passage through the $\mathrm{G}_{1} / \mathrm{S}$ checkpoint in human cancer cells. This is compatible with previous studies showing that blocking the canonical Ras signaling pathway - and therefore MAPK activation - in thyroid, melanoma, and hepatocellular carcinoma cell lines led to a similar block in the $\mathrm{G}_{1}$ phase of the cell cycle (10-12).

We confirmed the effects of MyD88 inactivation on cell proliferation with several established human cancer cell lines. The lung adenocarcinoma cell lines H358, H1975, and A549, and the colon cancer line $\mathrm{CaCo} 2$ all exhibited reduced proliferation upon treatment with MyD88-specific siRNA (Supplemental Figure 5).

Erk-interacting proteins such as the MAPK kinases MEK1/2 interact with Erk via a conserved $(\mathrm{K} / \mathrm{R})_{1-3}-\mathrm{X}_{1-5}-\phi \mathrm{X} \phi$ motif called $\mathrm{D}$ domain, where $\phi$ is a hydrophobic residue, typically Leu, Ile, Val, or Met (13). Interestingly, the MyD88 protein sequence contains such a motif (RRRLSLFL, aa 30-37) in its death domain that has been conserved throughout evolution (Supplemental Figure 6), suggesting a possible physical interaction between the 2 proteins. Indeed, as shown in Figure 2A, WT MyD88 (Flag-MyD88 WT), but not MyD88, where the arginines in the putative docking motif were changed into methionines (Flag-MyD88 R/M), coprecipitates with Erk. Interestingly, MyD88 did not interact with MEK under the same immunoprecipitation conditions (Figure 2B). Importantly, using the highly sensitive in situ proximity ligation assay (14), we show a time-dependent interaction between endogenous MyD88 and p-Erk upon activation of the Ras pathway (Figure 2C and Supplemental Figure 7).

To investigate the mechanistic basis of MyD88 participation in Erk activation, we hypothesized that MyD88 could interfere with MKP3-mediated Erk dephosphorylation. Indeed, MKP3, which is the major Erk-specific phosphatase involved in functional Erk downregulation, also binds to Erk via the same conserved $\mathrm{D}$ domain present in MyD88 (13). As shown in Figure 2D, MyD88 is capable of preventing MKP3-mediated Erk dephosphorylation, presumably through steric competition, thus maintaining Erk in an active, phosphorylated state. One could conversely expect that in absence of MyD88, MKP3 would have an easier access to p-Erk after activation. Indeed, MKP3 downregulation by RNA interference in 

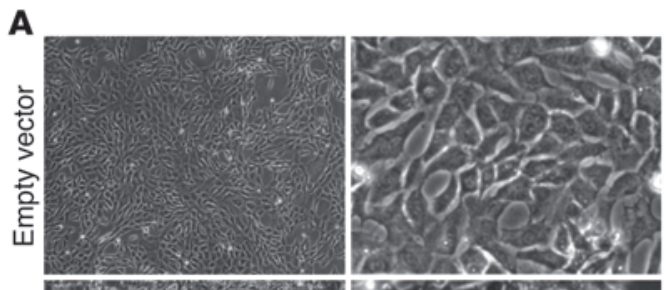

B

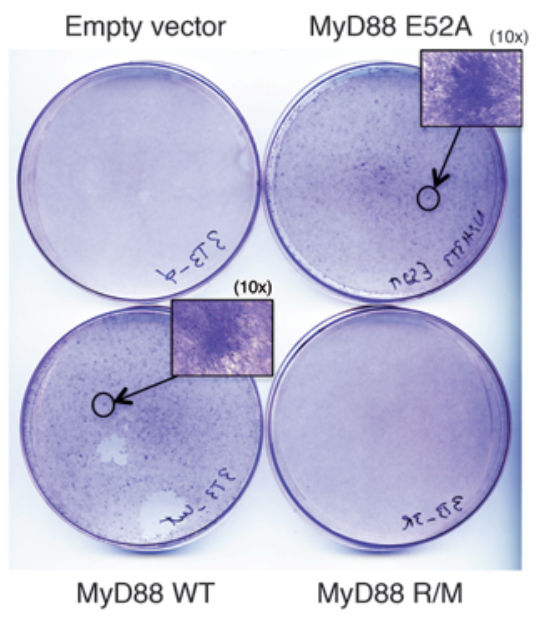

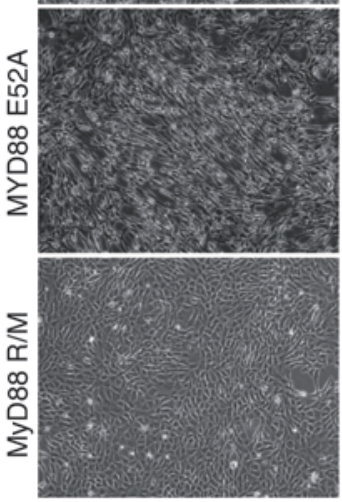

$(10 x)$

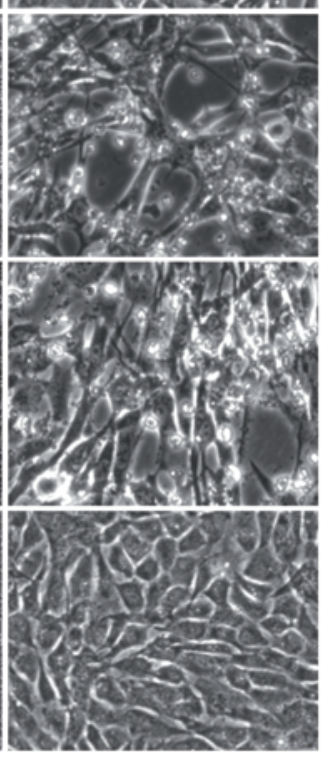

(40x)

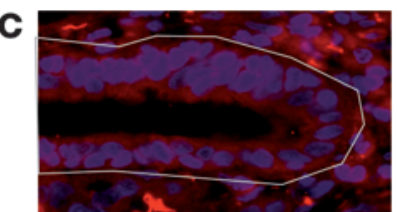

E

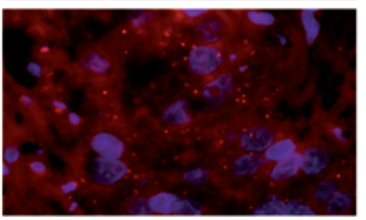

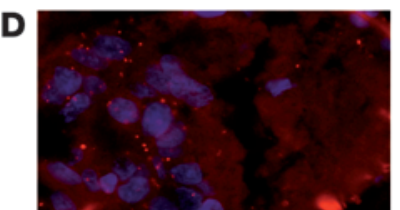

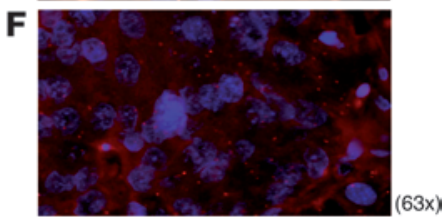

Figure 3

MyD88 induces Erk-dependent transformation of fibroblasts in vitro and interacts with p-Erk in primary human cancers. (A) NIH 3 T3 fibroblasts were transfected with Myc and either empty vector, MyD88 WT, MyD88 R/M, or MyD88-E52A. Antibiotic-selected MyD88 WT- and MyD88-E52A-, but not MyD88 R/M-transfected cells started showing signs of microscopic morphological transformation after 10 days of culture and (B) focus formation 3 weeks after culture. (C-F) MyD88 and p-Erk interaction in primary lung tumor tissue revealed by proximity ligation assay (red dots). Paraffin sections were stained with antibodies to MyD88 and p-Erk followed by the appropriate DNA-linked secondary antibodies according to the Duolink protocol. Shown are normal peritumoral epithelium (within the white outline) (C) and glandular tumor structures (D-F) from the same section.

MyD88-/- MEFs led to the restoration of full Erk phosphorylation (Figure 2E). Collectively, these results show that the reciprocal regulation of Erk phosphorylation by MyD88 and MKP3 may represent one way of regulating the canonical Ras/MAP kinase pathway.

Signaling molecules are activated to exert their biological effects either by posttranslational modifications (e.g., phosphorylation, ubiquitination, etc.) or via an increase in their protein levels. We investigated the consequences of MyD88 overexpression on the canonical MAPK pathway. Supplemental Figure 8 shows that in HeLa cells cultured in the presence of serum, MyD88 enhances MEK/Erk-dependent transcription of Elk-1 - which is presumably driven by growth factors present in the culture medium and/or by oncogenes that are active in the cell line - while the MyD88 mutant that cannot interact with Erk (MyD88 R/M) is unable to increase the activation of the canonical Ras/MAPK pathway, indicating that enhanced Erk activation by MyD88 is dependent on the physical interaction between the 2 proteins. Since, as shown above, MyD88 overexpression leads to a robust activation of the canonical Ras pathway, we asked whether MyD88 overexpression can lead to cell transformation. We cotransfected WT or mutant MyD88 with Myc in NIH-3T3 cells, selected for transfected cells with antibiotics, and looked for transformation.
As can be seen in Figure 3, MyD88 induces, in association with Myc, morphological transformation and focus formation in NIH-3T3 cells. Importantly, the MyD88 mutant that is unable to interact with Erk (MyD88 R/M), but not that which is unable to interact with IRAK (MyD88-E52A), lost the ability to participate in cell transformation, demonstrating that MyD88 has a cell-autonomous role in transformation through its interaction with Erk, and not through the TLR/IL-1R cell-signaling pathway.

We then examined the expression of MyD88 in human primary cancer tissues. In tumor sections from cancer patients, we found that MyD88, which exhibited a faint, cytoplasmic staining in peritumoral epithelial cells, was highly overexpressed in stomach $(n=5)$, colon $(n=5)$, and lung $(n=5)$ cancerous tissue in situ (Supplemental Figure 9). A heterogeneous overexpression of MyD88 was also observed in papillomas from DMBA/TPA-treated WT mice (Supplemental Figure 10). Since, as shown above, MyD88 is involved in Erk activation and cell transformation, we asked whether MyD88 and p-Erk interact in cancer cells in situ. Indeed, using the proximity ligation assay, we showed interactions between MyD88 and p-Erk in primary human cancer tissue (Figure 3, D-F), but not in adjacent normal peritumoral epithelium (Figure 3C). 
Previous reports showed that MyD88 is necessary for the protumorigenic inflammatory response $(4,5)$. In this study we have demonstrated what we believe is a novel, cell-autonomous role for MyD88 in cell transformation through its interaction with Erk and its involvement in its activation. Overall, our data are compatible with a scenario whereby in response to growth factors or oncogenic signaling, Erk is phosphorylated then rapidly dephosphorylated by MKP3. MyD88 binds to p-Erk via its $\mathrm{D}$ domain, thereby preventing the $\mathrm{p}$-Erk-MKP3 interaction via the same motif and maintaining Erk in an active, phosphorylated state for a longer period of time. At the same time, MyD88 allows the activation of NF- $\kappa$ B by soluble mediators (e.g., IL-1), leading to the activation of further inflammatory and mitogenic signals. This combination of MyD88-dependent signals leads to enhanced cell activation, proliferation, and eventually, transformation. (Supplemental Figure 11). This enhanced activation is likely to be due to prolonged baseline Erk phosphorylation resulting from decreased dephosphorylation. However, this may not be the only mechanism at work. It is indeed conceivable that MyD88 overexpression could induce, through an as-yet-undetermined mechanism, a quantitatively more potent activation of Erk, which would contribute to the ability of overexpressed MyD88 to induce Erk-dependent cell transformation. Further elucidation of the multiple facets of MyD88 activity, in particular at the interface between inflammation and cell transformation, should yield a clearer understanding of the converging signaling pathways that ultimately lead to cancer development.

\section{Methods}

Mice. Eight-week-old C57BL/6 (Charles River), MyD88-/- (ref. 15; a gift from S. Akira, Osaka University, Osaka, Japan) and $I L-1 R^{-/-}$mice (ref. 16; Jackson Laboratory) were used. Both gene-targeted strains were backcrossed into the C57BL/ 6 background for at least 9 generations. All animal studies were approved by the Schering-Plough Research Institute Ethics Committee, Dardilly, France.

In vivo carcinogenesis. Mice were treated with a single application of $25 \mu \mathrm{g}$ DMBA (Sigma-Aldrich) in $200 \mu \mathrm{l}$ acetone, followed by biweekly applications of $200 \mu \mathrm{l}$ of TPA (Sigma-Aldrich) of $10^{-4} \mathrm{M}$ solution in acetone for 20 weeks. Mice were visually inspected twice per week and tumors were counted.

1. West AP, Koblansky AA, Ghosh S. Recognition and signaling by toll-like receptors. Annu Rev Cell Dev Biol. 2006;22:409-437.

2. Lee MS, Kim YJ. Signaling pathways downstream of pattern-recognition receptors and their cross talk. Annu Rev Biochem. 2007;76:447-480.

3. Clevers H. At the crossroads of inflammation and cancer. Cell. 2004;118(6):671-674.

4. Rakoff-Nahoum S, Medzhitov R. Regulation of spontaneous intestinal tumorigenesis through the adaptor protein MyD88. Science. 2007; 317(5834):124-127.

5. Swann JB, et al. Demonstration of inflammationinduced cancer and cancer immunoediting during primary tumorigenesis. Proc Natl Acad Sci U S A. 2008;105(2):652-656.

6. Bizub D, Wood AW, Skalka AM. Mutagenesis of the Ha-ras oncogene in mouse skin tumors induced by polycyclic aromatic hydrocarbons. Proc Natl Acad Sci US A. 1986;83(16):6048-6052.
Transformation assays. MEFs were transfected with Ha-Ras ${ }^{\mathrm{v} 12}$ (Upstate) and c-Myc (a gift from J. Vlach, Schering-Plough, Kenilworth, New Jersey, USA), or an empty vector. 24 hours later, cells were plated, allowed to grow for 21 days, fixed, and stained with crystal violet. NIH 3 T3 fibroblasts were transfected with Myc and either empty vector, MyD88 WT, MyD88 R/M, or MyD88-E52A, selected with G418, and allowed to grow for 21 days.

$M A P K$ activation assay. MEFs were starved overnight, then treated with FGF8b (R\&D Systems). Erk phosphorylation and total Erk were evaluated by Western blot using anti-p-Erk (Sigma-Aldrich) and anti-Erk (Cell Signaling) antibodies, respectively.

Proximity ligation assay. Cells were stimulated with $10 \%$ serum for 5 and 10 minutes, fixed with $4 \%$ PFA, and permeabilized and blocked with $0.3 \%$ saponin, $10 \%$ BSA in PBS. Cells were then incubated with primary antibodies to MyD88 (Assay Designs) and p-ERK (Sigma-Aldrich), then with the appropriate, DNA-linked secondary antibodies, analyzed with the Duolink assay according to the manufacturer's instructions. The same protocol was applied to histological tumor sections.

MKP3/MyD88functional competition. Cells were transfected with pMKP-3-Myc $(2 \mu \mathrm{g})$ and/or pCMV-flag and/or PCMV-MyD88-flag (1 $\mu \mathrm{g})$. 24 hours after transfection, cells were starved for 20 hours followed by incubation with $30 \%$ FCS for 3 minutes. Cells were lysed and immunoblotted with the following antibodies: anti-p-ERK and anti-Erk (Cell Signaling Technology), anti-Flag (Sigma-Aldrich), and anti-Myc (BioMol).

Statistics. Statistical significance was evaluated using a 2-tailed Student's $t$ test. $P<0.0005$ was considered significant. All bar graph data shown represent mean \pm SEM.

\section{Acknowledgments}

We thank F. Berger, S. Isaac, and J.Y. Scoazec (Hospices Civils de Lyon [HCL], Lyon, France) for providing human tumor samples; P. Hainaut is supported by the Institut National du Cancer (INCa) and by the Ligue Contre le Cancer. T. Renno is supported by the Agence Nationale de la Recherche (ANR), the Association pour la Recherche sur le Cancer (ARC), and the Ligue Contre le Cancer.

Received for publication February 24, 2010, and accepted in revised form July 16, 2010.

Address correspondence to: Toufic Renno, CNRS UMR5201, Centre Léon Bérard, 28 rue Laënnec, 69373, Lyon, France. Phone: 33.469.166.629; Fax: 33.469.166.660; E-mail: renno@lyon.fnclcc.fr.
7. Burns K, Janssens S, Brissoni B, Olivos N, Beyaert $\mathrm{R}$, Tschopp J. Inhibition of interleukin 1 receptor/ Toll-like receptor signaling through the alternatively spliced, short form of MyD88 is due to its failure to recruit IRAK-4. J Exp Med. 2003;197(2):263-268.

8. Loiarro $\mathrm{M}$, et al. Identification of critical residues of the MyD88 death domain involved in the recruitment of downstream kinases. J Biol Chem. 2009;284(41):28093-28103.

9. Sumimoto H, Imabayashi F, Iwata T, Kawakami Y. The BRAF-MAPK signaling pathway is essential for cancer-immune evasion in human melanoma cells. J Exp Med. 2006;203(7):1651-1656.

10. Smalley KS, Eisen TG. Farnesyl transferase inhibitor SCH66336 is cytostatic, pro-apoptotic and enhances chemosensitivity to cisplatin in melanoma cells. Int J Cancer. 2003;105(2):165-175.

11. Weisenauer CA, Yip-Schneider MT, Wang Y, Schmidt CM. Multiple anticancer effects of blocking MEK-ERK signaling in hepatocellular carci- noma. J Am Coll Surg. 2004;198(3):410-421.

12. Ouyang B, et al. Inhibitors of Raf kinase activity block growth of thyroid cancer cells with RET/PTC or BRAF mutations in vitro and in vivo. Clin Cancer Res. 2006;12(6):1785-1793.

13. Sharrocks AD, Yang SH, Galanis A. Docking domains and substrate-specificity determination for MAP kinases. Trends Biochem Sci. 2000;25(9):448-453.

14. Söderberg O, et al. Direct observation of individual endogenous protein complexes in situ by proximity ligation. Nat Methods. 2006;3(12):995-1000.

15. Kawai T, Adachi O, Ogawa T, Takeda K, Akira S. Unresponsiveness of MyD88-deficient mice to endotoxin. Immunity. 1999;11(1):115-122.

16. Horai R, et al. Production of mice deficient in genes for interleukin (IL)-1alpha, IL-1beta, IL-1alpha/beta, and IL-1 receptor antagonist shows that IL-1beta is crucial in turpentine-induced fever development and glucocorticoid secretion. J Exp Med. 1998;187(9):1463-1475. 